\title{
Quantum transport of vortices in a weakly dissipative ring threaded by an Aharonov-Casher flux: A tight-binding model
}

\author{
Jian-Xin Zhu \\ Texas Center for Superconductivity, University of Houston, Houston, Texas 77204-5932 \\ and Department of Physics, University of Hong Kong, Pokfulam Road, Hong Kong \\ Z. D. Wang \\ Department of Physics, University of Hong Kong, Pokfulam Road, Hong Kong
}

(Received 27 May 1997)

\begin{abstract}
Based on the tight-binding model and taking into account the effect of dissipation as well as the disorder, we study quantum interference via the Aharonov-Casher effect for fluxons in a ring-shaped superconductor. The electrical resistance in terms of the transmission probability for a quantum vortex to traverse the ring is calculated. It is shown that a quantum interference effect is exhibited in the resistance in the presence of weak dissipation. Our analysis may also be applied to a Josephson-junction array system. In particular, by including the disorder effect, we are able to explain the experimental measurements in the dissipative regime done by Elion et al. [Phys. Rev. Lett. 71, 2311 (1993)].
\end{abstract}

[S0163-1829(97)52042-X]

In 1984 it was shown by Aharonov and Casher $^{1}$ (AC) that a neutral particle carrying a magnetic moment may exhibit a quantum-mechanical interference effect, which is a dual of the Aharonov-Bohm ${ }^{2}(\mathrm{AB})$ effect in which a charged particle is deflected in the presence of a vector potential even when the force exerted on the particle is absent. This AC effect has also been discussed for fluxons (magnetic vortices) in a type-II superconductor ${ }^{3-6}$ and for vortices in a ring-shaped two-dimensional array of Josephson junctions in the absence of dissipation. ${ }^{7}$ Recently, Elion et al. ${ }^{8}$ observed quantum interference of vortices in a Josephson-junction array, which may be again a manifestation of the AC effect. As pointed out by these authors, however, the phase-interference oscillation was observed in the flux-motion resistance. According to the conventional wisdom, the dissipation should destroy the phase-interference effect, and so Elion and co-workers' experiment presents a rather unique and challenging problem, ${ }^{8}$ which, to the best of our knowledge, has not been tackled seriously. In this paper, by taking into account the dissipation effect, we study the quantum coherence of fluxons via the AC effect in a ring-shaped type-II superconductor or a Josephson-junction array. It is shown that the interference could survive in the presence of weak dissipation. In addition, the disorder and the temperature effects have also been discussed. A satisfactory interpretation for the interference oscillation of the resistance observed in the experimental measurement by Elion et al. ${ }^{8}$ is presented.

Figure 1 shows schematically a ring-shaped type-II superconducting thin film lying in the $x y$ plane. This ring is of inner radius $r_{2}$ and outer radius $r_{1}$ and thickness $d$. The width of the ring arm, $r_{1}-r_{2} \ll r_{2}$, is comparable to the penetration depth. Each branch of the ring is attached to a type-I superconducting film, characterized by a critical magnetic field $H_{c}^{I}$, and they in turn are coupled to the driving current and voltage contacts. A straight line charge with charge density $\Lambda$ pierces perpendicularly the center of the ring, which creates a radial Coulomb electric field, $\mathcal{E}=(2 \Lambda / r) \hat{\mathbf{r}}$. A constant external magnetic field $H\left(H_{c 1}<H<H_{c}^{I}<H_{c 2}\right)$ is applied perpendicular to the $x y$ plane such that fluxons, each of which carries a quantized flux $\Phi_{0}=h c / 2 e$ and has a direction along the line segment that traces out the normal core of the fluxon, are induced in the mixed-state regime. Under this arrangement, we assume that the fluxon moves only in the azimuthal direction along the fixed contour of radius $r_{a}$ if a driving current is introduced. When considered in its essential nature as a topological excitation, a fluxon (vortex line) can be regarded as a quantum particle (boson) at extremely low temperatures. In particular, these fluxons or vortices in

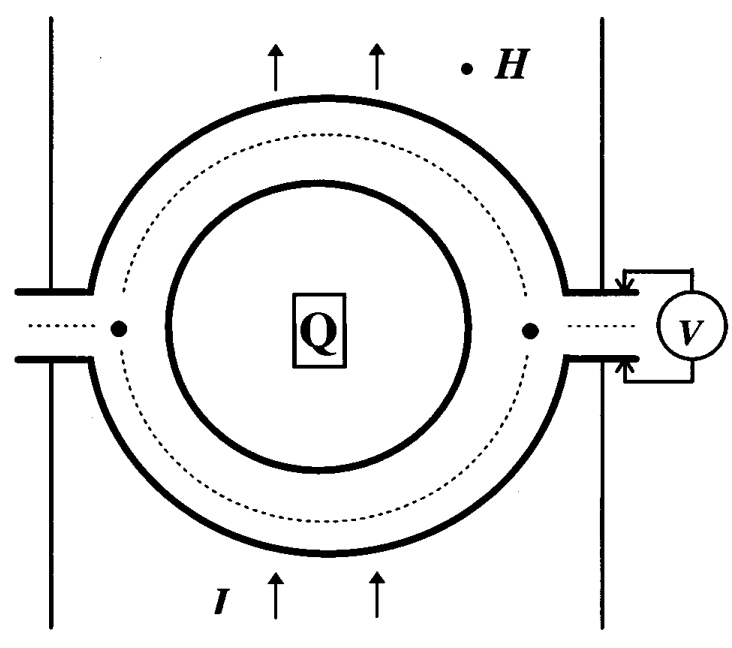

FIG. 1. Schematic drawing of a ring-shaped type-II superconductor. The fluxons are induced by a magnetic field. A straight charge line with charge density $\Lambda$ passes through the center of the ring, and $Q=\Lambda d$ where $d$ is the thickness of the ring. The dashed lines picture two trajectories traveled by fluxons. 
type-II superconductors or Josephson-junction arrays have infinite repulsive on-site interaction, leading to the hard-core constraint. Therefore, they can be treated as standard neutral hard-core bosons carrying an effective magnetic moment $\boldsymbol{\mu}$ $=\left(\Phi_{0} d / 4 \pi\right) \hat{\mathbf{z}}$, where $\hat{\mathbf{z}}$ is the unit vector orthogonal to the $x y$ plane. On the other hand, it is still a macroscopic object in all other respects. We are therefore in a position to study the dynamics of a macroscopic quantum particle, which is influenced by its interaction with the environment. The effective model Hamiltonian of the system plus environment may be written as

$$
H=H_{\mathrm{S}}+H_{\mathrm{R}}+H_{\mathrm{I}} \text {. }
$$

Here

$$
H_{\mathrm{S}}=\sum_{i} \epsilon_{i} b_{i}^{\dagger} b_{i}-\sum_{i j} V_{i j} b_{i}^{\dagger} b_{j}
$$

is the Hamiltonian of the isolated fluxon system. $\epsilon_{i}$ are the on-site energies, which take the value of zero on the two connected leads and are distributed randomly in an interval $[-W / 2, W / 2]$ on the ring. The hopping integrals in the leads are taken to be $V_{i j}=V$ for nearest-neighbor sites and zero otherwise. In the presence of an external electric field generated by the straight line charge, it has been indicated by Reznik and Aharonov ${ }^{3}$ that, although this electric field may be screened in the superconductor, there is still some part of the electric field penetrating the superconductor, which is able to make a flux, after traversing one round along the ring, and acquire a correct geometric phase, $\varphi_{\mathrm{AC}}$ $=(\hbar c)^{-1} \oint d \mathbf{l} \cdot(\mathcal{E} \times \boldsymbol{\mu})=2 \pi Q / Q_{0}$, with $Q=\Lambda d$ and $Q_{0}$ $=2 e .{ }^{9}$ As a consequence, the nearest-neighbor hopping integrals in the ring take the form $V_{i j}=V e^{i \Delta \theta_{i j}}$, where $\Delta \theta_{i j}$ is the phase acquired by the quantum vortex hopping from one site to the nearest-neighbor one. Since the AC effect is a global effect, ${ }^{3}$ it follows that $\Sigma \Delta \theta_{i j}=2 \pi Q / Q_{0}$, where the summation runs over all of the links on the ring. $b_{i}^{\dagger}$ and $b_{i}$ create and annihilate one fluxon on the $i$ th site, they commute on different sites

$$
\left[b_{i}^{\dagger}, b_{j}^{\dagger}\right]=\left[b_{i}, b_{j}\right]=\left[b_{i}^{\dagger}, b_{j}\right]=0, \quad(i \neq j),
$$

but anticommute on the same site (hard core constraint)

$$
\left\{b_{i}^{\dagger}, b_{i}\right\}=1, \quad\left\{b_{i}^{\dagger}, b_{i}^{\dagger}\right\}=\left\{b_{i}, b_{i}\right\}=0 .
$$

As we have shown in Ref. 5 a gas of $N_{b}$ hard-core bosons defined on the ring is equivalent to a gas of $N_{f}=N_{b}$ free fermions except for the introduction of a global phase factor $\exp \left[i \pi\left(N_{f}-1\right)\right]$ into the boundary condition obeyed by the fermion wave function. Since here we are only interested in the transport properties of the fluxon system, this global phase shift has no effect. ${ }^{10}$ After such a mapping, we may have

$$
H_{\mathrm{S}}=\sum_{i} \epsilon_{i} f_{i}^{\dagger} f_{i}-\sum_{i j} V_{i j} f_{i}^{\dagger} f_{j}
$$

where $f_{i}^{\dagger}$ and $f_{i}$ are the usual fermion operators defined on the lattice that satisfy the anticommutating relation

$$
\left\{f_{i}, f_{j}^{\dagger}\right\}=\delta_{i j}, \quad\left\{f_{i}, f_{j}\right\}=\left\{f_{i}^{\dagger}, f_{j}^{\dagger}\right\}=0 .
$$

In the eigenenergy representation, the Hamiltonian of the fluxon system then becomes $H_{\mathrm{S}}=\Sigma_{k} E_{k} f_{k}^{\dagger} f_{k}$, where $f_{k}^{\dagger}$ and $f_{k}$ are the fermion operators defined in the energy eigenstate space. The environment Hamiltonian is modeled by the harmonic oscillators, and in second quantization can be written as

$$
H_{\mathrm{R}}=\sum_{q} \omega_{q} a_{q}^{\dagger} a_{q}
$$

where $a_{q}^{\dagger}$ and $a_{q}$ are creation and annihilation operators of collective excitions which inelastically scatter the transporting quantum vortex. We use the independent boson model ${ }^{11}$ to describe the coupling between the quantum vortex system and the environment

$$
H_{\mathrm{I}}=\sum_{k, q} M_{k, q} f_{k}^{\dagger} f_{k}\left(a_{q}+a_{q}^{\dagger}\right)
$$

where $M_{k, q}$ is a set of coupling constants. Although this type of the coupling Hamiltonian is a bit ad hoc, the advantage lies in that with Eq. (5) the system plus the environment can be solved exactly. As we are mainly concerned with a qualitative description of transport properties of quantum vortices with dissipation, we are content to use this simplified coupling. Using this coupling Hamiltonian, one can find ${ }^{12}$ from the linear response Kubo formula in combination with the diagonalization technique developed by Mahan ${ }^{11}$ that the effective transmission probability in the presence of dissipation is

$$
\begin{aligned}
\mathcal{T}= & \beta \int d E_{1} d E_{2} f_{\mathrm{FD}}\left(E_{1}\right)\left[1-f_{\mathrm{FD}}\left(E_{2}\right)\right] \\
& \times \operatorname{Re}\left[t\left(E_{1}\right) t^{*}\left(E_{2}\right)\right] P\left(E_{1}, E_{2}\right)
\end{aligned}
$$

where $\beta$ is the inverse temperature, the Fermi-Dirac distribution function $f_{\mathrm{FD}}(E)=\left\{\exp \left[\beta\left(E-E_{F}\right)\right]+1\right\}^{-1}$, the transmission amplitude $t(E)$ is quantum mechanically calculated from the quantum vortex system $H_{\mathrm{S}}$ given by Eq. (3), $P\left(E_{1}, E_{2}\right)$ is the coupling function between the system and the environment and, if all collective modes have the same energy $\omega_{0}$ (Einstein model), is given by

$$
\begin{aligned}
P\left(E_{1}, E_{2}\right)= & \sum_{m=-\infty}^{\infty} \delta\left(E_{2}-E_{1}+m \omega_{0}\right) \\
& \times \exp \left[-\left(2 n_{\mathrm{BE}}+1\right) g\right] \\
& \times \exp \left(m \beta \omega_{0} / 2\right) I_{m}\left(2 \sqrt{n_{\mathrm{BE}}\left(n_{\mathrm{BE}}+1\right)} g\right),
\end{aligned}
$$

with $\omega_{0}$ the frequency of the collective modes, $I_{m}(x)$ is the Bessel function of complex argument, and $n_{\mathrm{BE}}=\left[\exp \left(\beta \omega_{0}\right.\right.$ $-1]^{-1}$ the distribution function of the collective modes, the dimensionless coupling constant $g=\Sigma_{q}\left\langle\left(M_{k, q}\right.\right.$ $\left.\left.-M_{k^{\prime}, q}\right)^{2}\right\rangle_{k, k^{\prime}} / \omega_{0}^{2}$. Evidently, in the limit of $g=0, P\left(E_{1}\right.$ $\left.-E_{2}\right)$ goes to $\delta\left(E_{1}-E_{2}\right)$ and Eq. (6) reduces to the nondissipation case. In particular, we wish to point out that Eq. (6) is derived in the single-particle approximation [see Eq. (3)]. For the present system, if the density of vortices is not so high, this approximation appears to be acceptable as the di- 


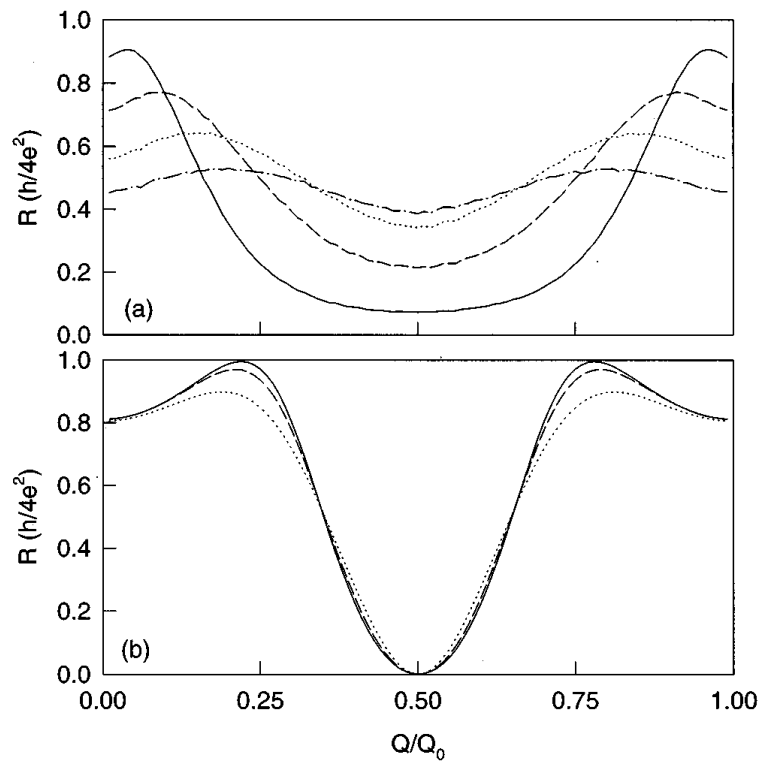

FIG. 2. (a) The zero-temperature vortex-motion resistance as a function of $Q / Q_{0}$ with $E_{F} / V=0$ and $g=0$ for four different values of the disorder strength, $W / V=1$ (solid line), 2 (dashed line), 3 (dotted line), 4 (dashed-dotted line). (b) The disorder-free vortexmotion resistance as a function of $Q / Q_{0}$ with $E_{F} / V=0.5, g=0$ for three different values of temperature, $\beta V=50$ (solid line), 20 (dashed line), 10 (dotted line).

rect interaction between two vortices at different sites is too weak to be included in the Hamiltonian.

On the experimental aspect, the measured voltage across the gate of width $W_{a}$ at the endpoint $B$ is the line integral of the electric field, $\mathcal{V}=\int \mathbf{E} \cdot d \mathbf{l}$. For the case considered here, the contribution to the integral from the electric field created by the line charge can be neglected, and the only contribution comes from the type-II superconducting region. Consequently, $\mathcal{V} \approx E W_{a}$, where $E$ is the electric field induced by the fluxon motion. This electric field is given by ${ }^{13}$

$$
\mathbf{E}=\frac{1}{c}(\mathbf{b} \times \mathbf{v})=\frac{n_{a} \Phi_{0}}{c}(\hat{\mathbf{z}} \times \mathbf{v}),
$$

where $\mathbf{b}$ is the magnetic induction, $\mathbf{v}$ is the velocity of the fluxon, and $n_{a}$ the area density of fluxons. By taking into account the elastic and phase-breaking scattering of the fluxons, the measured voltage becomes $\mathcal{V}=\left(\Phi_{0} / c\right) \delta n_{l} v \mathcal{T}$, where $\delta n_{l}$ is the number of states for fluxons per unit length in the energy interval $\delta \epsilon$ and $\mathcal{T}$ is the transmission probability given by Eq. (6). Due to the Lorentz force, the energy imbalance is given by $\delta \epsilon=\Phi_{0} \mathcal{I} / c$, where $\mathcal{I}$ is the driving current. In view of the fact that we have a one-dimensional density of states, $\partial n_{l} / \partial \epsilon=1 / h v$, we obtain the resistance ${ }^{6}$

$$
R=\frac{\mathcal{V}}{\mathcal{I}}=\frac{h}{(2 e)^{2}} \mathcal{T}
$$

This formula relates the quantum vortex-motion resistance to the transmission probability of a vortex, in which the quantum-mechanical interference effect and the dissipation effect are essentially included.
In the following, we would like to calculate the transmission probability within the tight-binding model given by Eq. (3). So far, pioneered by Imry, Büttiker, Gefen, and Azbel, ${ }^{14-16}$ the studies of the conductance behavior of a mesoscopic electronic ring enclosing a magnetic flux are mainly based on the continuum model and a clear physical understanding for the problem has been given. For our purpose, we use instead the tight-binding model to study the quantum transport of the vortex motion. In this model, the disorder and the band effects can be taken into account conveniently. ${ }^{17}$ Suppose a vortex impinging from the lefthand side (LHS) leads into the left junction, the Bloch state on the LHS is $\psi_{n}=\exp (i k n a)+r \exp (-i k n a), \quad n=0,-1$, $-2, \ldots$, where $r$ is the reflection amplitude and $k$ is the longitudinal wave vector satisfying the dispersion relation $\epsilon$ $=-2 \mathrm{~V} \cos (k a)$ ( $a$ being the vortex lattice constant). A scattering state on the right-hand side (RHS) for a vortex impinging from the LHS is given by $\psi_{n}=t \exp (i k n a), n$ $=0,1,2, \ldots$ By substituting $1+r$ and $t$ for the wave function amplitudes on the opposite junctions connecting the leads and the ring, and solving Eq. (3) to eliminate the wave function amplitudes at the sites forming the ring, we find analytically the transmission amplitude for an eight-site ring to be

$$
t=\frac{2 i e^{-i \varphi / 2} \sin (k a) D_{0}}{D_{1} e^{-2 i k a}+D_{2} e^{-i k a}+D_{3}},
$$

where

$$
\begin{gathered}
D_{0}=X^{(u)} X^{(d)}\left(\epsilon_{2}^{(u)}+\epsilon_{2}^{(d)} e^{i \varphi}\right)+e^{i \varphi} X^{(u)} Y^{(d)} Z^{(d)} \\
+X^{(d)} Y^{(u)} Z^{(u)} \\
D_{1}=X^{(u)} X^{(d)} \\
D_{2}=X^{(u)}\left(Z^{(d)}-Y^{(d)}\right)+X^{(d)}\left(Z^{(u)}-Y^{(u)}\right), \\
D_{3}=\left(X^{(d)}+X^{(u)} e^{-i \varphi}\right)\left(\epsilon_{2}^{(u)}+\epsilon_{2}^{(d)} e^{i \varphi}\right) \\
+\left(Z^{(d)}-Z^{(u)} e^{-i \varphi}\right)\left(Y^{(d)} e^{i \varphi}-Y^{(u)}\right), \\
X^{(u, d)}=\epsilon_{3}^{(u, d)}\left(\epsilon_{2}^{(u, d)} \epsilon_{1}^{(u, d)}-1\right)-\epsilon_{1}^{(u, d)}, \\
Y^{(u, d)}=\epsilon_{2}^{(u, d)} \epsilon_{1}^{(u, d)}-1, \\
Z^{(u, d)}=1-\epsilon_{3}^{(u, d)} \epsilon_{2}^{(u, d)}
\end{gathered}
$$

and $\epsilon_{i}^{(u, d)}, i=1,2,3$, are the energies of three sites on the upper and lower branch of the ring. Equation (10) shows clearly that the resistance will oscillate in the external charge $Q$ with the period $Q_{0}=2 e$. Although our calculations are carried out for an eight-site ring as an illustration, there should be no qualitative changes in the results when the ring size is varied.

We plot in Fig. 2(a) the vortex motion resistance at zero temperature as a function of the external charge for different values of the disorder strength $W$ but with the fixed Fermi energy $E_{F} / V=0$ and no dissipation $g=0$. Here $E_{F}=$ $-2 V \cos \left(k_{F} a\right)$ is the Fermi energy of fermions used to represent hard-core bosons, where $k_{F}=\pi \rho$ with $\rho$ the linear density of vortices. In our case, the disorder comes from the 
vortex pinning fluctuations. At weaker disorder, the resistance oscillates with the amplitude as large as $h /(2 e)^{2}$. As the disorder strength increases, the resistance oscillation decreases. In particular, the resistance maxima decrease while the resistance minima increase. Moreover, the positions of the resistance maxima changes with the increase of the disorder but those of the resistance minima are always fixed at $Q=Q_{0} / 2$. With such a feature, the resistance period halving may occur at some specific value of the disorder strength. As can be seen, in the strong disorder limit, the resistance is almost independent of the change of external charge via the $\mathrm{AC}$ effect, and approaches a nonzero constant value. Figure 2(b) shows the disorder-free vortex-motion resistance as a function of the external charge for different values of the temperature $T$ but with the fixed Fermi energy $E_{F} / V=0.5$ and no dissipation. As one can see, the resistance oscillation amplitude decreases with the increase of the temperature. In contrast to the disorder case, this decrease of the resistance oscillation is uniquely due to the reduction of the resistance maxima with the increase of the temperature. As long as the ring is ordered, the resistance always vanishes when the external charge is at $Q=Q_{0} / 2$ regardless of the temperature.

In Fig. 3(a) we plot the disorder-free vortex-motion resistance as a function of the external charge against different values of the dissipation strength. In the calculation, $\beta \omega_{0}$ $=0.5$ is taken so that there are thermally activated collective modes available to scatter the quantum vortex. In the weak dissipation limit, $g / \beta \omega_{0} \leqslant 1$, which is physically reasonable. In addition, $E_{F} / V=0.5$ and $\beta V=10^{3}$ are taken. Comparing Fig. 2(b) with Fig. 3(a), we can see clearly that the increase of dissipation decreases the amplitude of the resistance as a whole, showing the similar effect of the temperature. This resemblance between the two effects demonstrates, at least in the weak dissipation limit, that the phase-breaking time is determined by both the temperature and the coupling between a quantum system and the environment. In Fig. 3(b), we plot the vortex motion resistance as a function of the external charge by taking $W / V=2$ and $g=0.2$ to include both the disorder and the dissipation effects. The values of other parameters are the same as those for Fig. 3(a). As seen
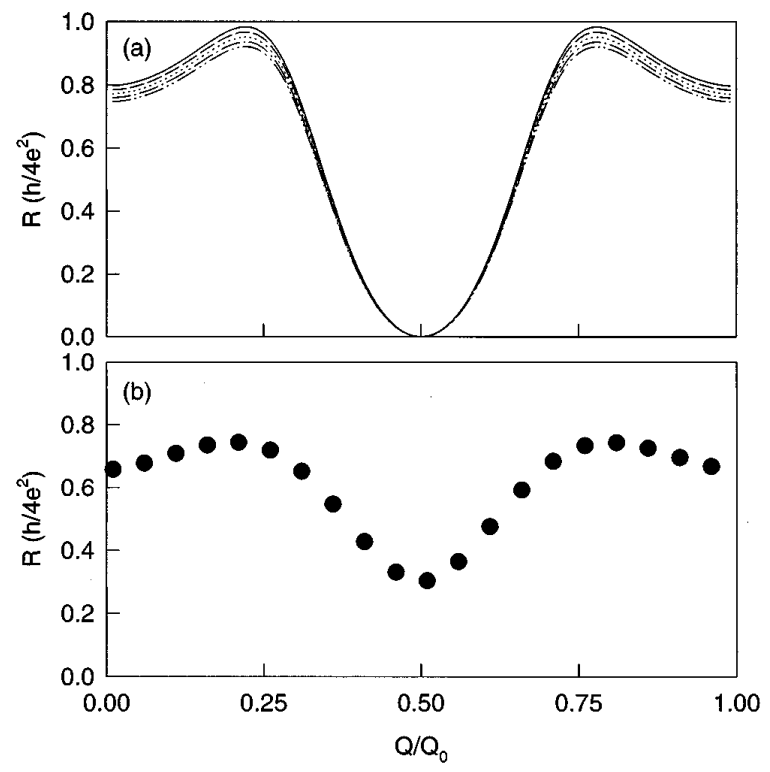

FIG. 3. (a) The disorder-free vortex-motion resistance as a function of $Q / Q_{0}$ for five different values of the dissipation strength, $g=0.1$ (solid line), 0.2 (dashed line), 0.3 (dotted line), 0.4 (dasheddotted line), 0.5 (dashed-double-dotted line). (b) The vortex-motion resistance versus $Q / Q_{0}$ with $W / V=2$ for a typical value of the dissipation strength $g=0.2$. Here $E_{F} / V=0.5, \beta V=10^{3}, \beta \omega_{0}$ $=0.5$.

from Fig 3(b), by taking into the disorder effect, we can explain essentially the experimental measurement by Elion et al. ${ }^{18}$ in the dissipative regime since our analysis may also be applied to the ring of a Josephson-junction array. As for fluxons in a ring-shaped type-II superconductor as we propose in Fig. 1, the transmission probability of the fluxon traversing the system may be smaller, but the full AC effect is still expected to be observable in a very clean sample.

This work was supported by the RGC grant of Hong Kong under Grant No. HKU262/95P and the Texas Center for Superconductivity at the University of Houston.
${ }^{1}$ Y. Aharonov and A. Casher, Phys. Rev. Lett. 53, 319 (1984).

${ }^{2}$ Y. Aharonov and D. Bohm, Phys. Rev. 115, 485 (1959).

${ }^{3}$ B. Reznik and Y. Aharonov, Phys. Rev. D 40, 4178 (1989).

${ }^{4}$ Jian-Xin Zhu, Z. D. Wang, and Qin-Wei Shi, J. Phys. A 27, L875 (1994).

${ }^{5}$ Z. D. Wang and Jian-Xin Zhu, Phys. Rev. B 52, 5275 (1995).

${ }^{6}$ Z. D. Wang, Jian-Xin Zhu, and Q. Wang, J. Phys. Soc. Jpn. 65, 3398 (1996).

${ }^{7}$ B. J. van Wees, Phys. Rev. Lett. 65, 255 (1990); T. P. Orlando and K. A. Delin, Phys. Rev. B 43, 8717 (1991).

${ }^{8}$ W. J. Elion, J. J. Wachters, L. L. Sohn, and J. E. Mooij, Phys. Rev. Lett. 71, 2311 (1993).

${ }^{9}$ If the neglected small Hall force is of the form $\alpha \mathbf{v} \times \hat{\mathbf{z}}$ and can be treated quantum mechanically, there may be the small fluctuation of the Hall-force-induced phase shift, but it is usually negligible when compared with the AC phase under study.

${ }^{10}$ The phase shift due to the connection between the ring and the leads is also neglected.
${ }^{11}$ G.D. Mahan, Many-Particle Physics (Plenum Press, New York, 1990).

${ }^{12}$ S. Feng, Phys. Lett. A 143, 400 (1990).

${ }^{13}$ Y. B. Kim and M. J. Stephen, in Superconductivity, edited by R. D. Parks (Marcel, New York, 1969); Z. D. Wang, Jinming Dong, and C. S. Ting, Phys. Rev. Lett. 72, 3875 (1994).

${ }^{14}$ R. Landauer, Philos. Mag. 21, 863 (1970); M. Büttiker, Phys. Rev. Lett. 57, 1761 (1986).

${ }^{15}$ Y. Gefen, Y. Imry, and M. Ya. Azbel, Phys. Rev. Lett. 52, 129 (1984).

${ }^{16}$ M. Büttiker, Y. Imry, and M. Ya. Azbel, Phys. Rev. A 30, 1982 (1984).

${ }^{17}$ O. Entin-Wohlman, C. Hartzstein, and Y. Imry, Phys. Rev. B 34, 921 (1986); D. Kowal, U. Sivan, O. Entin-Wohlman, and Y. Imry, ibid. 42, 9009 (1990).

${ }^{18}$ In their experiment, the periodicity of the single electron charge $e$ (i.e., $-e / 2 \leqslant Q<e / 2$ ) is due to quasiparticle tunneling in the presence of Coulomb blockade. 